\title{
Anticipating the Societal Challenges of Nanotechnologies
}

\author{
Diana M. Bowman • Elen Stokes • \\ Michael G. Bennett
}

Received: 7 February 2013 / Accepted: 14 February 2013 / Published online: 14 March 2013

(C) Springer Science+Business Media Dordrecht 2013

\begin{abstract}
In this article we sketch out the landscape for this Special Issue on anticipating and embedding the societal challenge of nanotechnologies. Tools that actors may choose to employ for these processes are articulated, and further explored through the introduction of the seven articles which comprise this Issue. Taken together, these articles create a cogent narrative on the societal challenges posed by nanotechnologies. They are drawn together by three distinct themes, each of which is briefly considered within this context of this Introductory article".
\end{abstract}

Keywords Societal challenges $\cdot$ Regulatory inheritance $\cdot$ Law $\cdot$ Loci of influence

Attempts to anticipate both the positive and negative effects of a technological change are not unique to

D. M. Bowman $(\bowtie)$

Risk Science Centre and Department of Health

Management and Policy, School of Public Health,

The University of Michigan,

1420 Washington Heights,

48109 Ann Arbor, MI, USA

e-mail: dibowman@umich.edu

E. Stokes

Cardiff Law School, Cardiff University,

Wales, UK

e-mail: stokeser@cf.ac.uk modern society. The invention of the printing press, commonly attributed to Johannes Gutenburg, in the mid-15th century resulted in radical and at the time unprecedented social and economic change [1, 3]. The mass technological innovation underpinning the Industrial Revolution resulted in a reordering of society, bringing with it extraordinary and farreaching impacts $[10,13]$. So, too, did the invention and mass production of, for example, the motor vehicle, the aeroplane and the mobile phone. Proponents and opponents of these technologies can be found at all stages of research, development and commercialization, as too can lively debate on how best to maximize the social benefits of change while minimizing the threat of potential harm. The difference perhaps nowadays, compared with the premodern era, is that we are growing ever more attuned to, and able to perceive, the consequences of

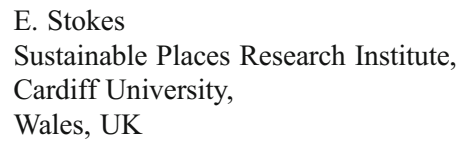

M. G. Bennett

Northeastern Law School,

Boston, MA, USA

e-mail: M.Bennett@neu.edu 
technological advances. We also have, by virtue of technological change, more opportunities to participate in such debates, and voice our concerns.

The tools with which societal actors, including industry and government, estimate, articulate and mediate the likely impact of technological change have matured alongside the technologies themselves. Today, these tools include constructive technology assessment (CTA) [7, 16], socio-technical scenario methods and strategy articulation [4, 14], up-stream engagement [18], de facto assessments [16], foresighting $[2,11]$ and anticipatory governance $[8$, 9]. As further evidence that the management of technological development is high on policy agendas, governments have invested in institutions designed to support such practices, as illustrated by the United Kingdom's Foresight Programme and Singapore's Strategic Futures Network [6, 12].

There can be little doubt that the emergence of socially disruptive technologies, including nanotechnologies, cognitive technologies and synthetic biology, require critical short, medium and longer-term thinking about how their products and applications are conceptualized, understood and organized in society. The challenges are complex and ubiquitous in nature, and as illustrated by the case of nanotechnologies, something that all governments must grapple with. And some jurisdictions, to put it bluntly, will do it better than others.

This Special Issue draws together seven contributions, each of which has its genesis within large Dutch funded programs designed to promote the responsible development of nanotechnologies. The scope of these contributions, however, lies beyond the Netherlands and are relevant to a broader audience. In the first article, "Bridging the Gap between innovation and ELSA: the TA program in the Dutch nano-R\&D program NanoNed", Rip and van Lente trace the history of societal contestations around technologies, and the Dutch Government's attempts to address the challenges posed by advances in, for example, biotechnologies and more recently nanotechnologies. The lessons from such exercises, which are both positive and negative, are discussed by the two eminent scholars. They note that "reflexive co-evaluation [must be] more than public and stakeholder participation", and that one of the key tasks is the development of an "understanding the dynamics of nanotechnology development and its embedding in society".
In the second article "Context Matters: Promises and Concerns regarding nanotechnologies for water and food application," Kulve et al. employ what they call "de facto assessment" techniques in order to explore specific implications, social acceptance and discourse in two important areas of technology application. By examining the framing of nanotechnologies within the scholarly literature related to food and water sciences the authors describe how assessments of the technology's promises, deliverables and implications are hermeneutically contingent and complex. Framing, they argue based on their exploratory study, is shaped by "specific discursive repertoires, historical experiences with new technologies and specific business models and concerns". In this respect, the article highlights the fundamental importance of excavating the relationships between nanotechnologies - its meaning(s) and governance-and the diverse range of stakeholders.

The third article in this Special Issue builds on the notion of scientific discourse, and the need to find common ground in a dynamic environment. Consensus assumes a greater importance, but is notoriously more difficult to achieve in an environment characterized by high levels of uncertainty and contestation. In "Wide Reflective Equilibrium as a normative model for responsible governance", Doorn examines the functionality of two models derived from policy theory that may be employed for framing the public policy challenges posed by nanotechnologies. The aim, as she states, is "to find a consensus on (a framework for) policy measures and regulation". This is no easy task, especially given the distinct "lack of knowledge, [has ensured that] many of the early discussions on the ethical aspects of nanotechnology have a strong futuristic and sciencefictional overtone". Both models examined are underpinned by an ethos of consensus on which policies may then be progressively built and refined. Doorn highlights the need for establishing order and creating common futures between multiple actors and agendas in order to advance beyond the level of abstraction and develop concrete policy measures.

This theme of actors and agendas, and the challenges thereof, is further developed by Krabbenborg in her article "DuPont and Environment Defense Fund co-construction a risk framework for nanoscale materials: An occasion to reflect on interaction processes in a joint inquiry". Krabbenborg frames her analysis of this high-profile collaboration using a moral labor 
lens. Drawing upon a series of interviews, the article examples "to what extent "symmetry" occurred in the interactions and what were the enabling and constraining conditions" that fostered the partnership. While acknowledging the need for early involvement of all stakeholders in the identification and management of issues posed by emerging technologies, Krabbenborg argues that while efforts to encourage and/or foster joint inquiries between civil society actors and industry are admirable, an inherent asymmetry will exist within the partnership; they are founded on, and rely upon, an unworkable and unsustainable division of moral labour between policy "outsiders" (civil society) and "insiders" (experts, decision-makers).

In the fifth paper, "Meta-regulation and nanotechnologies: The challenge of responsibilisation with the European Commission's Code of Conduct for Responsible Nanosciences and Nanotechnologies Research", Dorbeck-Jung and Shelly-Egan examine the efficacy of meta-regulation as a means of balancing the underlying and competing tensions in nanotechnology politics and policies. The inherent conflict between economic, social and public health goals underpins their analysis, and highlights the trade-offs that are made within the political sphere accordingly. As articulated by the authors, "there is an inherent temporal dilemma in the governance and regulation of nanoscience and nanotechnologies: regulation too soon may potentially stifle innovation and cut off the emergence of beneficial technologies, while a 'wait and see' approach to regulation may lead to a catastrophe which could have been anticipated or dealt with more effectively". The authors, through their examination of the European Union Code of Conduct, capture the desire to think reflexively about regulation, so that it is not just nanotechnologyenabled products, but the process of nanotechnology regulation itself, that become regulated.

This chimes with a general theme of the Special Issue-that the task of "regulating nanotechnologies" involves more than simply regulation of the technology itself. It is, rather, an inherently more complex and rigorous process, that must be refined in order to reflect the increasing sophistication of the field. Moreover, it involves forging and regulating relationships, regulating regulation, regulating the loci of decisionmaking influence and control, and regulating visions of the past, present and of "responsible" futures.
The penultimate article, by Kica and Bowman, "Transnational Governance Arrangements: Legitimate Alternatives to Regulating Nanotechnologies?", examines the role that standard setting bodies have in underpinning research and development activities, the commercialization of nanotechnologies and the regulatory arrangements. It explores an important strategy for responsive meta-regulation, and the role that one body in particular, the International Organization for Standardization (ISO) Technical Committee 229, is playing in the promulgation of voluntary standards. While such standards are developed to meet the needs of the client-i.e. industry and not government regulatory agencies - such measures receive broad transnational support. However, as Kica and Bowman point out, the "pay-to-play" approach of ISO creates barriers of entry and raises several challenges to legitimacy that should not be overlooked.

Reichow and Dorbeck-Jung, in the final article, examine an issue that while not unique to nanotechnologies, is further exacerbated by it-compliance with voluntary regulation. In "Discovering specific conditions for compliance with soft regulation related to work with nanomaterials", the authors focus specifically on the occupational health and safety context. Here, the authors note that while in many jurisdictions employers have a "legal obligation to care for safe workplaces", the lack of scientific and regulatory guidance creates ambiguity in the nano-context. There is, as they note, a paucity of data and "risk assessment and risk management are extremely difficult tasks since there is limited evidence about the risks of nanomaterials". In exploring three specific instruments that have been developed to assist employers fulfil their legal obligations, the authors suggest that willingness to comply with soft instruments is only part of the picture. Availability, and appropriateness of technological devices, expertise and methods, may also limit a party's ability to comply. Accordingly, questions of regulating nanotechnologies-including a firms' willingness to voluntary submit to additional compliance hurdles-cannot easily be answered without looking carefully at the context, conditions and constraints.

Taken together, these seven articles create a cogent narrative on the societal challenges posed by nanotechnologies. Three key themes draw the articles together: that new technologies present fresh opportunities to prise open and re-examine what were 
previously settled debates; that regulating technologies may be premised on technical and scientific debates, but in reality are underpinned by regulating relationships; and that the law, whether it be public or private, national or transnational, is omnipresent in framing these debates. As lawyers, we would argue that this role is often overlooked.

Drawing on the first theme, the emergence of new technologies allows society to reflect, and challenge, the status quo. Or at least it should. There is now a significant body of literature that articulates the economic and societal opportunities offered by nanotechnologies $[15,17]$. Less has, however, been said about the opportunities that disruptive technologies, including nanotechnologies and other emerging technologies, provide for society to re-examine existing structures, instruments, decision-making apparatus and narratives. To these ends, we argue that new technologies do not so much present opportunities for entirely new rules but instead should force us to reflect on existing provisions and practices.

The second theme, regulating relationships, is implicit within each of the seven articles presented in this Special Issue. Since Fielder and Reynolds [5] first raised questions relating to the regulation of nano-based products and processes, the regulatory debate has primarily been framed in the context of scientific uncertainty, the need for data-driven assessment techniques, and risk management. Despite the billions of dollars that has now been spent on addressing scientific questions, ambiguity still remains. To a large extent, the debate around the technology is still framed in this way.

What is emphasised within the context of this Special Issue is that aside from the methodological and epistemological problems associated with technological novelty, nanotechnologies produce an unrealistic set of assumptions about the relationship between science and society, experts and publics, and upstream commitments and downstream outcomes. Even if they do not warrant an overtly 'new' regulatory approach, the emergence of a disruptive technology such as nanotechnologies and synthetic biology, offers the opportunity to reflect more systematically upon how our assumed future (a technologically innovative and responsible one) comes to be defined and projected through legal and policy arrangements. These issues entail less transient questions of hazard exposure and risk, and more fundamental yet more intractable questions of who should decide propositional truths about future development and technological trajectories, how those decisions ought to be made, and using what conceptual framework. Nanotechnologies bring these fundamental unresolved tensions and instabilities into sharp focus, and help to break open the entrenched, culturalinstitutional ideologies of new technology regulation. Recent efforts to promote responsible innovation highlight, among other things, that it is not just technological products and processes that need regulating; technological cultures need renegotiating and regulating too.

A further consequence of the dominant discourse, which to date has focused almost exclusively on the absence of, and contradictory nature of, scientific knowledge and evidential requirements in law, is that legal intervention is treated more cautiously in situations of profound uncertainty. At least in most jurisdictions, most of the time. It may be with good reason that the introduction of legislative and regulatory provisions expressly addressing nanotechnologies is postponed until such measures may be said to be based on sound science and are accepted by the scientific community at large. However, the interaction between law and knowledge can be subtler and is often non-linear. It is not the case that the role of the law is limited unless, and until, further evidence of harm can be produced. As these articles show, the law has an important part to play in establishing order and clarity, generating further information, and positioning loci of influence, control and responsibility. It also creates a level playing field. It may be the case, then, that we turn to law notwithstanding problems of ambiguity and complexity.

In drawing to a close this Introduction, we would like to express our gratitude to Professor John Weckert, Editor-in-Chief of NanoEthics, for his ongoing support. This Issue represents Professor Weckert's last at the helm, and we are delighted to be able to bid him adieu and to thank him, on behalf of the academic community, for his invaluable contribution to scholarly debates in the field. We would also like to thank our colleagues and contributors to this Special Issue, and the NanoNextNL program for its continuing support.

\section{References}

1. Dittmar JE (2011) Information technology and economic change: the impact of the printing press. Q J Econ 126 (3):1133-1172 
2. Doubleday R (2007) Risk, public engagement and reflexivity: alternative framings of the public dimensions of nanotechnology. Health Risk Soc 9(2):211-227

3. Eisenstein EL (1979) The printing press as an agent of change. CUP, Cambridge

4. Elzen B, Geels FW, Hofman PS, Green K (2004) Sociotechnical scenarios as a tool for transaction policy: an example from the traffic and transport domain. In: Boelie E, Geels FW, Green K (eds) System innovation and the transition to sustainability: theory, evidence and policy. Edward Elgar, Cheltenham, p 251

5. Fiedler FA, Reynolds GH (1994) Legal problems of nanotechnology: an overview. South Calif Interdiscip Law J 3:593-629

6. Foresight (2012) Annual review 2011. HM Government, London

7. Genus A, Coles AM (2005) On constructive technology assessment and limitations on public participation in technology assessment. Technol Anal Strat Manag 17(4):433-443

8. Guston D (2008) Innovation policy: not just a jumbo shrimp. Nature 454:940-941

9. Guston D, Sarawitz D (2002) Real-time technology assessment. Technol Soc 24(1-2):93-109

10. Hobsbawm EJ, Wrigley C (1999) Industry and empire: the birth of the industrial revolution. Penguin, New York
11. Johnston R (2001) Foresight-refining the process. Int J Technol Manag 21(7):711-725

12. Kuosa T (2012) The evolution of strategic foresight: navigating public policy making. Gower, Surrey

13. Landes DS (2003) The unbound Prometheus: technological chance and industrial development in western Europe from 1750 to the present. CUP, Cambridge

14. Rip A, te Kulve H (2008) Construction technology assessment and socio-technical scenarios. In: Fisher E, Selin C, Wetmore JM (eds) The yearbook of nanotechnologies in society, vol 1. Springer, New York, pp 49-70

15. Roco MC, Harthorn B, Guston D, Shapira P (2011) Innovative and responsible governance of nanotechnology for societal development. In: Roco MC et al. Nanotechnology research directions for societal needs in 2020: retrospective and outlook. Science Policy Reports, 1, 561-617

16. Schot J, Rip A (1997) The past and future of constructive technology assessment. Technol Forecast Soc Chang 54 (203):251-268

17. Seear K, Petersen A, Bowman DM (2010) Communicating with citizens about nanotechnologies: views of key stakeholders in Australia. Monash University and The University of Melbourne, Melbourne

18. Wilsdon J, Wills R (2004) See-through science: Why public engagement needs to move upstream. Demos, London 